\title{
Síntomas digestivos y respuesta clínica en lactantes con alergia a la proteína de leche de vaca
}

\author{
JOSÉ FERNANDO VERA CH. ${ }^{1}$, ANDREA RAMÍREZ V. ${ }^{2}$
}

1. Gastroenterólogo, Nutriólogo, Epidemiólogo clínico. Sección de Gastroenterología, Hepatología y Nutrición Pediátrica. Hospital Universitario Fundación Santa Fe de Bogotá. Profesor Universidad de los Andes. Grupo de Investigación PEDIAFE. Bogotá DC, Colombia.

2. Médico, Hospital Universitario Fundación Santa Fe de Bogotá. Estudiante Maestría en Salud Pública, Universidad de los Andes. Grupo de Investigación PEDIAFE, Centro de Investigación en Salud (CEIS), Hospital Universitario Fundación Santa Fe de Bogotá. Bogotá DC, Colombia.

\begin{abstract}
Gastrointestinal symptoms and clinical response of infants with cow's milk protein allergy

Introduction: Quality of life and nutritional status of infants with cow's milk protein allergy (CMA) can be compromised without a proper diagnosis and treatment. Objective: To describe digestive symptoms, family and perinatal history and nutritional status of $\leq 12$ month infants with CMA between June 2007 and August 2011. Patients and Method: A retrospective and descriptive study in $\leq 12$ month old infants suspecting CMA was performed. Family history, perinatal and clinical characteristics were studied. Cases without food test or open challenge test, or whose reason for consultation corresponded to other pathology were excluded. The program STATA 11.1 was used for statistical analysis and $\mathrm{p}<0.05$ was considered statistically significant. Results: 40 infants ( $57.5 \%$ boys), age $3 \pm 0.4$ months and history of $2 \pm 0.3$ months presenting the following: 20 of them (50\%), vomiting/regurgitation; 8 (20\%), bloody diarrhea; 4 (10\%) failure to thrive; 4 (10\%), colic/irritability and bloody normal deposition was present in 3 individuals (7.5\%). Family and perinatal history: 35 (87.5\%) were bottle-fed during the first week of life; $31(77.5 \%)$ had $\geq 30$ year old mothers; 28 (70 \%) described 2 family members (parents/siblings) with atopy; 27 (67.5\%) were delivered via cesarean. At the $14 \pm 1.4$-month follow-up, 19 (47.5\%) responded to extensively hydrolyzed formula \pm breastfeeding; 19 to amino acid formula \pm breastfeeding and one patient to breastfeeding exclusively. The $Z$-score for P/E was: $-1.05 \pm 1.28 ; \mathrm{T} / \mathrm{E}:-0.96$ \pm 1.60 ; BMI: $-0.61 \pm 1.03$ in the first consultation; after a year, scores improved: P/E: $-0.15 \pm 1.24$; T/E: -0.29 \pm 1.14 and BMI: $-0.04 \pm 1.20(\mathrm{p}<0.05)$, independent from formula $(\mathrm{p}=\mathrm{NS}) .23(74 \%)$ tolerated whole milk when reached a year old. Conclusions: In this study, the predominant early symptoms were vomiting, regurgitation and bloody diarrhea. Among the antecedents, early exposure to cow's milk, parental atopy and cesarean delivery were described. With proper treatment, nutritional recovery was observed independent from formula used, and cow's milk tolerance was observed in a high percentage of infants when they reached a year old.

(Key words: Food allergy, cow's milk protein, nutrition, infant).

Rev Chil Pediatr 2013; 84 (6): 641-649
\end{abstract}

Recibido el 2 de septiembre de 2012, devuelto para corregir el 29 de octubre de 2012, segunda versión 14 de noviembre de 2012, tercera versión 18 de julio de 2013, aceptado para publicación el 7 de octubre de 2013.

Este estudio se realizó en el Hospital Universitario Fundación Santa Fe de Bogotá Colombia. No hubo financiamiento a través de grants o convocatorias.

Este trabajo cumple con los requisitos sobre consentimiento /asentimiento informado, comité de ética, financiamiento, estudios animales y sobre la ausencia de conflictos de intereses según corresponda. 


\section{RESUMEN}

Introducción: La calidad de vida y estado nutricional del lactante con alergia a la proteína de leche de vaca (APLV) pueden comprometerse sin un adecuado diagnóstico y tratamiento oportuno. Objetivo: Describir síntomas digestivos, antecedentes familiares y perinatales, estado nutricional y respuesta de lactantes $\leq 12$ meses con APLV entre junio de 2007 y agosto de 2011. Pacientes y Método: Estudio retrospectivo, descriptivo en lactantes $\leq 12$ meses de edad que consultan por sospecha de APVL. Se investigaron antecedentes familiares, perinatales y características clínicas. Se excluyó a los casos sin prueba de alimentación o provocación abierta, o cuyo motivo de consulta correspondió a otra patología. Se usó el programa STATA especial edición 11,1 para el análisis estadístico. Se consideró estadístico y significativo un $\mathrm{p}<0,05$. Resultados: Se diagnosticaron 40 lactantes (57,5\% varones), edad 3,0 $\pm 0,4$ meses, clínica de $2 \pm 0,3$ meses de: vómito/regurgitación: 20 , diarrea sanguinolenta: 8 , falla en el crecimiento: 4, cólico/irritabilidad: 4, deposición normal con sangre en 3 . Los antecedentes familiares y perinatales fueron ingesta de biberón en la $1^{\mathrm{a}}$ semana de vida: 35 , madre $\geq 30$ años: 31 casos, atopia en 2 familiares (padres/hermanos): 28 , parto por cesárea: 27 . Al seguimiento de $14 \pm 1,4$ meses, 19 respondieron a fórmula ampliamente hidrolizada \pm seno; 19 a fórmula de aminoácidos \pm seno y un paciente lactancia exclusiva. El Z-score de P/E:-1,05 $\pm 1,28$; T/E: $-0,96 \pm 1,60$; IMC: $-0,61 \pm 1,03$ en primera consulta, mejoró al año: P/E: $-0,15 \pm 1,24$; T/E: $-0,29 \pm 1,14$ e IMC: $-0,04 \pm 1,20(p<0,05)$, independiente de la fórmula $(\mathrm{p}=\mathrm{NS})$. Veintitres toleraron leche entera al año. Conclusiones: En este estudio los síntomas predominantes al inicio de los pacientes con APLV fueron vómitos, regurgitación y diarrea sanguinolenta. Entre los antecedentes destacan la exposición temprana a PLV, atopia familiar y parto por cesárea. Con el tratamiento adecuado hubo recuperación nutricional independientemente de la fórmula usada y tolerancia a PLV al año en un alto porcentaje de lactantes.

(Palabras clave: Alergia alimentaria, Proteína de leche de vaca, Nutrición, Lactante).

Rev Chil Pediatr 2013; 84 (6): 641-649

\section{Introducción}

La alergia a la proteína de leche de vaca (APLV) constituye la alergia alimentaria más frecuente en el primer año de vida, y la razón fundamental es que la proteína de la leche de vaca (PLV) suele ser la primera proteína a la cual se enfrentan los niños con lactancia materna o sin ella ${ }^{1,2}$. El problema radica en que el crecimiento y desarrollo psicomotor pueden verse comprometidos sin un adecuado diagnóstico y tratamiento ${ }^{3,4}$. El diagnóstico de APLV es difícil, y no existen pruebas diagnósticas sensibles ni específicas especialmente en el primer año de vida, por lo que las dietas de eliminación y las pruebas de provocación son esenciales para el diagnóstico ${ }^{5}$. El cuadro clínico aunque es variable requiere un alto índice de sospecha ${ }^{6}$. No hay estudios suficientes en Colombia que muestren el comportamiento clínico ni la historia natural de la APLV en lac$\operatorname{tantes}^{3-8}$.

En la literatura se ha evidenciado un aumento de la incidencia de las alergias ali- mentarias especialmente en países desarrolla$\operatorname{dos}^{9,10}$, sin embargo, los estudios en Colombia también describen cifras alarmantes ${ }^{11-13}$. Se sabe que la prevalencia de APVL se encuentra entre el $2 \%$ y el $7,5 \%$ en la población menor de un año de edad y que debido a que no hay signos patognomónicos de la misma existe un sobrediagnóstico alcanzando una prevalencia hasta del $15 \% 5$.

La APLV se puede encontrar también en lactantes alimentados exclusivamente con leche materna, aunque la incidencia en este grupo es baja $(0,5 \%)$ y la presentación clínica no es tan severa ya que la concentración de la PLV en la leche materna es cien mil veces menor que en la leche de vaca ${ }^{14}$.

Las manifestaciones clínicas de la APLV se producen principalmente a nivel del tracto digestivo (50\%-60\%), la piel (50\%-60\%), y el tracto respiratorio $(20 \%-30 \%)$ y varían de intensidad de leve a moderada a grave ${ }^{14,15}$. Se ha descrito que en lactantes con antecedentes familiares y perinatales tales como tener padres atópicos y/o hermanos atópicos la prevalencia 
de APVL puede ser entre 40-60\%. Adicionalmente el riesgo de APVL se incrementa cinco veces al tener una madre atópica, tres veces al tener una madre mayor de 30 años de edad, tres veces al nacer por cesárea, diez veces al nacer por cesárea y tener una madre atópica; tres veces al recibir fórmula a base de PLV tempranamente y dos veces al haber recibido lactancia por corto tiempo ${ }^{16-25}$.

Ninguna de las pruebas diagnósticas disponibles en la rutina clínica prueba o excluye por completo la $\mathrm{APLV}^{7,26}$. Una buena historia clínica, incluyendo antecedentes familiares de atopia, antecedentes de madre fumadora ${ }^{17}$, hiperbilirrubinemia neonatal, aumento de la tripsina fecal $^{19}$, ser de género masculino ${ }^{25}$ y un examen clínico cuidadoso son la clave en el proceso de diagnóstico.

Las pruebas cutáneas (leche fresca o extractos de leche entera), de parche o IgE específicas indican sensibilización y no son necesariamente una prueba de reacción alérgica. La sensibilidad y especificidad de las pruebas cutáneas es de $31,8 \%$ y $90,3 \%$ y para IgE específica de $20,5 \%$ y $88,9 \%$, respectivamente ${ }^{27}$. Un estudio doble ciego controlado con placebo es la regla de oro en el diagnóstico de la APLV, pero en la práctica diaria sólo sé realiza un desafío, reto o provocación abierta ${ }^{5}$.

El objetivo del estudio fue describir síntomas digestivos, antecedentes familiares y perinatales, estado nutricional y respuesta de lactantes $\leq 12$ meses con sospecha de APVL a quienes se les realizó prueba de eliminación y de provocación abierta, en el período comprendido entre junio de 2007 y agosto de 2011.

\section{Pacientes y Método}

Estudio retrospectivo descriptivo de las características clínicas, manifestaciones digestivas, antecedentes familiares y perinatales en lactantes $\leq 12$ meses que consultaron con síntomas sugestivos de APLV a la sección de gastroenterología pediátrica del Hospital Universitario Fundación Santa Fe de Bogotá (HUFSFB) entre junio de 2007 y agosto de 2011.

Fueron incluidos en el estudio los pacientes que cumplieron los siguientes criterios: 1) te- ner una prueba de eliminación y provocación abierta presentando mejoría clínica con la administración de una fórmula extensamente hidrolizada -FEH- (Nutramigén $\left.{ }^{\circledR}\right)$, o a base de aminoácidos (Neocate $\left.{ }^{\circledR}\right)$, o a base de proteína de soya y/o suspensión de lácteos a la madre (prueba de eliminación); y 2) quienes posterior ( $\geq 4$ semanas del reto) a la reintroducción de una fórmula a base de PLV o ingesta de lácteos de la madre (prueba de provocación abierta) reprodujeron los síntomas digestivos por los que consultaron la primera vez. Se excluyeron historias clínicas de lactantes que no tuvieron prueba de eliminación y provocación abierta o que hubieran consultado por otras causas: síndrome de malabsorción, enfermedades metabólicas, hepáticas, renales, etc. ${ }^{1,3-8,10,16-}$ ${ }^{20,22,23,25,27-45}$.La Información de las historias clínicas, fue recolectada del sistema de información HIS-ISIS ${ }^{\circledR}$ del HU-FSFB y MEDSYS V4.0 ${ }^{\circledR}$ de la Asociación Médica de los Andes (AMA), en un cuestionario. Se realizó una prueba piloto en 10 pacientes. La información fue completada mediante llamada telefónica a los padres del paciente.

El estado nutricional para la primera, segunda y la consulta del año de edad fue calculado de acuerdo al z-score para peso/edad; talla/edad e IMC con el sofware: OMS Anthro versión 3.2.2. (2011). Los resultados fueron consignados en una base Excel 2010. Se realizaron pruebas de $\chi^{2}$ : para variables nominales y t- student en variables de distribución normal. Las diferencias fueron clasificadas como estadísticamente significativas cuando $p<0,05$. El análisis estadístico se realizó con el programa STATA Special Edition 11.1 (licensed \& serial number: 71606281563). El protocolo fue presentado al Centro de Estudios e Investigación en Salud (CEIS) y Comité de Ética e Investigaciones del HU-FSFB y fue aprobado.

\section{Resultados}

Se incluyeron 40 lactantes $(57,5 \%$ género masculino) que cumplieron los criterios de inclusión, de 3,0 $\pm 0,4$ meses de edad, con clínica de $2 \pm 0,3$ meses de vómito/regurgitación en $20(50 \%)$ pacientes; diarrea sanguinolenta 
Tabla 1. Estado nutricional (Z-score $\pm \mathrm{DS}^{1}$ ) de la primera consulta y al año de edad en lactantes con alergia a la proteína de leche de vaca

\begin{tabular}{|cccc|}
\hline Z-Score (DS) ${ }^{\mathbf{1}}$ & Primera consulta $\mathbf{n}=\mathbf{3 7}$ & Consulta 12 meses $\mathbf{~ = ~ 3 7}$ & $\mathbf{p}^{*}$ \\
\hline Peso/Edad & $-1,05 \pm 1,28$ & $-0,15 \pm 1,24$ & 0,0000 \\
\hline Talla/Edad & $-0,96 \pm 1,60$ & $-0,29 \pm 1,14$ & 0,0004 \\
\hline IMC $^{2}$ & $-0,61 \pm 1,03$ & $-0,04 \pm 1,20$ & 0,0022 \\
\hline
\end{tabular}

'DS = desviación estándar; ${ }^{2} \mathrm{IMC}=$ índice de masa corporal. * $\mathrm{p}<0,05$ (t-student muestras pareadas).

en $8(20 \%)$ pacientes; falla en el crecimiento en $4(10 \%)$ pacientes; cólico y/o irritabilidad en $4(10 \%)$ pacientes y deposición normal con sangre en $3(7,5 \%)$ pacientes. Se excluyeron 95 lactantes con sospecha de APLV y que no tenían la prueba de provocación abierta. El Z-score de los 40 pacientes fue: Peso/Edad: $-1,05 \pm 1,28$; Talla/Edad: $-0,96 \pm 1,60$ e índice de masa corporal (IMC): $-0,61 \pm 1,03$ en la primera consulta de gastroenterología pediátrica. A la consulta de seguimiento de gastroenterología pediátrica al año de edad asistieron 37 pacientes, quienes mejoraron nutricionalmente: Peso/Edad: $-0,15 \pm 1,24$; Talla/Edad: $-0,29$ $\pm 1,14$ e IMC: $-0,04 \pm 1,20,(\mathrm{p}<0,05)$ (tabla 1$)$. Seis $(15 \%)$ pacientes tenían Z-score de Peso/ Edad $<2$ desviaciones estándar (DS), 5 Z-score Talla/Edad $<2$ DS, 5 IMC/Edad $<2$ DS, y un paciente sobrepeso en la primera consulta.

Antes de la consulta de gastroenterología sus pediatras sospecharon el diagnóstico de APLV en $50 \%$ de los casos, sin embargo, a la mayoría les ordenaron cambio a fórmulas sin lactosa, soya, parcialmente hidrolizadas \pm lactosa e inclusive se les suspendió la lactancia materna a algunos. En cuanto a tratamiento farmacológico 14 pacientes recibieron procinéticos, 18 ranitidina, 8 inhibidores de bomba de protones y cinco antiácidos sin mejoría de los síntomas de vómito y regurgitación frecuentes. Tres pacientes recibieron antimicrobianos. Los 10 pacientes con vómito y regurgitación frecuentes, tenían radiografías de esófago, estómago y duodeno que descartaban anomalías anatómicas.

La frecuencia de factores que se asociaron a APLV en los 40 lactantes fueron: 1) ingesta de PLV ofrecida en biberón en la primera semana de vida en $35(87,5 \%)$ de los cuales 23 la recibieron antes de las $24 \mathrm{~h}$ de vida; 2 ) madre $\geq 30$ años en el momento del parto en 31 (77,5\%); 3 ) antecedente de atopia (asma, rinitis, dermatitis atópica) en dos familiares (padres y/o hermanos) en $28(70 \%)$; 4) parto por cesárea en $27(67,5 \%)$ de las cuales 6 fueron electivas; y $5)$ madre fumadora en $22(55 \%)$ pacientes.

En el seguimiento por gastroenterología pediátrica por $14 \pm 1,4$ meses, $19(47,5 \%)$ pacientes respondieron a una FHE \pm lactancia materna y a los 19 lactantes que no respondieron a una FEH y que continuaron con los síntomas iniciales después de un mes de tratamiento (falla en el tratamiento) se les suministró una fórmula a base de aminoácidos \pm lactancia materna y dieta sin PLV a la madre, presentando una mejoría clínica. Un paciente mejoró clínicamente con el uso de una fórmula de soya que había iniciado su pediatra y que fue usada durante el seguimiento por gastroenterología, y otro paciente mejoró con lactancia exclusiva realizando la madre una dieta libre de lactosa y suplementándose con calcio (tabla 2).

A tres pacientes se les realizó esofagogastroduodenoscopia y/o colonoscopia para descartar enfermedad celiaca y/o gastroenteropatía eosinofílica.

Se comparó la frecuencia de los factores de riesgo descritos en la literatura, en los 19

Tabla 2. Tratamiento en lactantes con alergia a la proteína de leche de vaca

\begin{tabular}{|lcc|}
\hline Tratamiento & $\mathbf{n}=\mathbf{4 0 *}$ & $\mathbf{\%}$ \\
\hline $\begin{array}{l}\text { Fórmula extensamente hidrolizada } \pm \\
\text { lactancia* }\end{array}$ & 19 & 47,5 \\
\hline Fórmula de aminoácidos \pm lactancia* & 19 & 47,5 \\
\hline Fórmula de soya \pm lactancia* & 1 & 2,5 \\
\hline Lactancia exclusiva* & 1 & 2,5 \\
\hline *Madre con dieta libre de lácteos y suplementada con calcio.
\end{tabular}


Tabla 3. Tratamiento y factores asociados con alergia a la proteína de leche de vaca

\begin{tabular}{|c|c|c|c|c|}
\hline Factor de Riesgo & $\begin{array}{l}\text { Tipo de Fórm } \\
\begin{array}{c}\text { Extensamente hidrolizada } \\
n=19\end{array}\end{array}$ & $\begin{array}{l}\text { Aminoácidos } \\
n=19\end{array}$ & OR (IC 95\%) & $\mathbf{p}^{*}$ \\
\hline Madre $\geq 30$ años & 15 & 16 & $0,7(0,90-4,0)$ & 0,67 \\
\hline Madre fumadora & 10 & 11 & $0,8(0,19-3,5)$ & 0,74 \\
\hline \multicolumn{5}{|l|}{ Atopia } \\
\hline 2 padres & 4 & 4 & $1,0(0,15-6,5)$ & 1,00 \\
\hline 1 padre +1 hermano & 6 & 7 & $0,8(0,17-3,7)$ & 0,73 \\
\hline 2 padres +1 hermano & 2 & 4 & $0,4(0,35-3,7)$ & 0,37 \\
\hline Parto por cesárea & 14 & 12 & $1,6(0,33-8,3)$ & 0,48 \\
\hline Biberón < 1 semana & 17 & 17 & $1,0(0,06-15,3)$ & 1,00 \\
\hline
\end{tabular}

Tabla 4. Cambio del estado nutricional (Z-Score) según fórmula usada como tratamiento en alergia a la proteína de leche de vaca

\begin{tabular}{|lccc|}
\hline Z-Score (DS ${ }^{\prime}$ ) & $\begin{array}{c}\text { Tipo de Fórmula } \\
\text { Extensamente hidrolizada } \\
\mathbf{n}=\mathbf{1 7}\end{array}$ & $\begin{array}{c}\text { Aminoácidos } \\
\mathbf{n}=\mathbf{1 8}\end{array}$ & $\mathbf{p}^{*}$ \\
\hline Peso/Edad & $-0,90 \pm 1,18$ & $-0,95 \pm 1,10$ & $0,89^{*}$ \\
\hline Talla/Edad & $-0,61 \pm 1,32$ & $-0,75 \pm 0,95$ & $0,71^{*}$ \\
\hline IMC $^{2}$ & $-0,64 \pm 1,03$ & $-0,53 \pm 1,24$ & $0,77^{*}$ \\
\hline
\end{tabular}

${ }^{1} \mathrm{DS}=$ desviación estándar; ${ }^{2} \mathrm{IMC}=$ índice de masa corporal. * $\mathrm{p}=\mathrm{NS}$ (t-student -grupos independientes).

pacientes que respondieron a una $\mathrm{FEH}$, con los 19 pacientes quienes no respondieron a una FEH y requirieron la introducción de una fórmula a base de aminoácidos, cuyo costo es cuatro veces mayor en el mercado colombiano y no se encontraron diferencias significativas (tabla 3).

Veintitres $23(74 \%)$ pacientes toleraron la PLV entre los 12 y los 17 meses, $13 \%$ entre los 18 y 23 meses y $13 \%$ después de los 2 años de edad.

Como síntomas asociados se determinó que $35 \%$ de los pacientes antes del año tenían episodios de broncoespasmo y necesidad de inhaladores, 52,5\% síntomas de rinitis y dermatitis atópica. El 67,5\% toleró el huevo y 72,5\% el pescado antes del primer año de vida.

Para identificar si había diferencias nutricionales entre la primera y la consulta del año de edad con el gastroenterólogo pediatra y el tipo de fórmula usada, se comparó el cambio nutricional entre los 17 pacientes que recibieron una FEH y los 18 que recibieron una fórmula a base de aminoácidos. No se encontraron diferencias significativas en Peso/ Edad $(\mathrm{p}=0,89)$; Talla/Edad $(\mathrm{p}=0,71)$ e IMC $(p=0,77)$, entre los dos grupos (tabla 4).

La tolerancia al pescado y huevo se presentó antes del año de edad en la mayoría de pacientes $(67,5 \%)$ y a la PLV entre los $12-17$ meses en el $74 \%$ de los pacientes, datos que no han sido reportados en nuestro país.

\section{Discusión}

La APLV es la alergia alimentaria más frecuente en primer año de vida.

Se requieren más estudios prospectivos en la región para desarrollar estrategias públicas de prevención en salud y diagnóstico temprano ${ }^{7,21,33,37,42,48,50,53-59}$.

Las formas de presentación clínica digestiva más frecuentes en este grupo de pacientes fueron vómito/regurgitación y la diarrea sanguinolenta en el $70 \%$ de los lactantes, lo cual está de acuerdo con otros estudios en Latino- 
américa $^{14,15}$ y en el resto del mundo $5,7,29,42,46,47$. Solamente en el $50 \%$ de los pacientes hubo sospecha de APLV por parte del pediatra y en la mayoría de pacientes se usaron conductas nutricionales inapropiadas tales como: el uso de otras fórmulas a base de PLV, soya, sin lactosa e inclusive la suspensión de la lactancia materna. Lo anterior habla de falta de información sobre las conductas nutricionales que se deben adoptar en estos $\operatorname{casos}^{5,7,8,42}$.

Los antecedentes familiares y perinatales asociados a APVL más frecuentes fueron: exposición temprana a la PLV en $87,5 \%$, que la madre tenga $\geq 30$ años en $77,5 \%$ e historia de atopia en dos familiares de primer grado en $70 \%$ de los pacientes.

La mayoría de estos pacientes fueron expuestos a la PLV en la primera semana de vida y $57,5 \%$ en las primeras $24 \mathrm{~h}$. Llama la atención, que el $67,5 \%$ de estos niños nacieron por cesárea, de las cuales el 22,2\% fueron electivas. Esta cifra obtenida en el estudio, representa más del doble de las cifras reportadas en Bogotá en el 2008, con un $36 \%$ de nacimientos por cesárea del total de partos registrados principalmente en instituciones privadas (veeduría Distrital de Bogotá DC) 3,5,8,17,18,21,22,28,42,48-52.

En cuanto al estado nutricional, la falla en el crecimiento $(<2 \mathrm{DS})$ en Peso/Edad en el $15 \%$ de los pacientes y el riesgo nutricional (-1-2 DS) en $32,2 \%$ es superior al encontrado en Brasil ${ }^{53}$. El déficit nutricional observado en el momento de la primera consulta puede ser atribuible, ya sea al uso inapropiado de sucedáneos de la leche de vaca, a un inicio tardío o duración insuficiente del tratamiento adecuado.

Estos pacientes mejoraron nutricionalmente al año de edad, independientemente de las fórmulas usadas como tratamiento $(p>0,05)$. Aunque no se encontraron diferencias en el estado nutricional ni en la frecuencia de factores de riesgo en este grupo de pacientes, las razones por las cuales algunos pacientes no respondieron a una $\mathrm{FEH}$ y requirieron tratamiento con una fórmula mucho más costosa a base de aminoácidos podría explicarse por la carga atópica, la severidad del cuadro antes del diagnóstico, los antecedentes familiares y los factores de riesgo alérgicos ${ }^{20-29}$.
Aunque los pacientes tuvieron una evolución clínica corta $(2 \pm 0,3$ meses $)$ antes de consultar al gastroenterólogo pediatra, entre el $35-52,5 \%$ de los pacientes presentaron síntomas de dermatitis atópica, asma y rinitis alérgica antes del año de edad. Esto habla de la importancia de hacer prevención primaria al fomentar la lactancia exclusiva, evitar el uso de fórmulas de PLV, y evitar las cesáreas innecesarias entre otros ${ }^{20-29}$.

Este estudio describe la información recolectada de las historias clínicas de un grupo de pacientes y analiza su comportamiento clínico en un período de tiempo, no cuenta con grupo control y el tamaño está dado por el número de pacientes disponibles que cumplieron los criterios de inclusión. Nuevos estudios prospectivos deben llevarse a cabo para desarrollar estrategias públicas de prevención en salud y diagnóstico temprano de esta entidad, especialmente en el grupo pediátrico ${ }^{7,21,33,37,42,48,50,53-59}$.

\section{Conclusión}

En este estudio clínico retrospectivo y descriptivo, que incluyó solamente lactantes que tenían mejoría de los síntomas con una prueba de eliminación y provocación abierta a las cuatro semanas (práctica diaria realizada en nuestro país), se documentó que los síntomas de vómito, regurgitación y diarrea sanguinolenta fueron los síntomas más frecuentes. La exposición temprana a la PLV, la atopia familiar y el parto por cesárea fueron los antecedentes predominantes, y los pacientes mejoraron con la suspensión de leche de vaca a la madre o fórmulas extensamente hidrolizadas. La mayoría de los pacientes toleró la introducción de leche de vaca entre los 12 a 18 meses de edad. Estos hallazgos son similares a otros estudios realizados en Latinoamérica.

\section{Agradecimientos}

Los autores de este trabajo agradecen su contribución y apoyo a los estudiantes de Medicina de la Universidad de los Andes: Juan Camilo Córdoba, Pablo Andrés Rodríguez y 
Hernán Nicolás Lemus y a los referencistas de la Biblioteca Satélite de Medicina de la Universidad de los Andes: Jenny Milena Machetá, Daniel Hernández y Luis Fernando Gómez.

\section{Referencias}

1.- Venter, Arshad SH: Epidemiology of food allergy. Pediatr Clin North Am 2011; 58 (2): 327-49, ix.

2.- Venter C, Pereira B, Grundy J, et al: Incidence of parentally reported and clinically diagnosed food hypersensitivity in the first year of life. The Journal of allergy and clinical immunology 2006; 117 (5): 1118-24.

3.- Keil T: Epidemiology of food allergy: what's new? A critical appraisal of recent population-based studies. Curr Opin Allergy Clin Immunol 2007; 7 (3): 259-63.

4.- Vieira MC, Morais MB, Spolidoro JV, et al: A survey on clinical presentation and nutritional status of infants with suspected cow' milk allergy. BMC Pediatr 2010; 10: 25.

5.- Vandenplas Y, Koletzko S, Isolauri E, et al: Guidelines for the diagnosis and management of cow's milk protein allergy in infants. Arch Dis Child 2007; 92 (10): 902-8.

6.- Noimark L, Cox HE: Nutritional problems related to food allergy in childhood. Pediatr Allergy Immunol 2008; 19 (2): 188-95.

7.- De Greef E, Hauser B, Devreker T, Veereman-Wauters $G$, Vandenplas $Y$ : Diagnosis and management of cow's milk protein allergy in infants. World J Pediatr 2012; 8 (1): 19-24.

8.- Vandenplas $Y$, Plaskie K: Safety and adequacy of an optimized formula for pediatric patients with cow's milk-sensitive enteropathy. Minerva Pediatr 2010; 62 (4): 339-45.

9.- Branum AM, Lukacs SL: Food allergy among U.S. children: trends in prevalence and hospitalizations. NCHS Data Brief 2008; 10: 1-8.

10.- Venter C, Pereira B, Grundy J, et al: Incidence of parentally reported and clinically diagnosed food hypersensitivity in the first year of life. J Allergy Clin Immunol 2006; 117 (5): 1118-24.

11.- Marrugo J, Hernández L, Villalba V: Prevalence of self-reported food allergy in Cartagena (Colombia) population. Allergol Immunopathol (Madr) 2008; 36 (6): 320-4.

12.- Sole D, Mallol J, Camelo-Nunes IC, Wandalsen GF: Prevalence of rhinitis-related symptoms in Latin American children-results of the International Study of Asthma and Allergies in Childhood (ISAAC) phase three.
Pediatr Allergy Immunol 2010; 21 (1 Pt 2): e127-36.

13.- Sole D, Mallol J, Wandalsen GF, Aguirre V: Prevalence of symptoms of eczema in Latin America: results of the International Study of Asthma and Allergies in Childhood (ISAAC) Phase 3. J Investig Allergol Clin Immunol 2010; 20 (4): 311-23.

14.- Marina O, Fernández A, Follett FR, et al: [Cow's milk protein allergy: proposed guidelines for the management of children with cow's milk protein allergy]. Arch Argent Pediatr 2009; 107 (5): 459-67.

15.- Correa FF, Vieira MC, Yamamoto DR, Speridiao Pda $G$, de Morais MB: Open challenge for the diagnosis of cow's milk protein allergy. J Pediatr (Rio J) 2010; 86 (2): 163-6.

16.- Bjorksten B: Genetic and environmental risk factors for the development of food allergy. Curr Opin Allergy Clin Immunol 2005; 5 (3): 249-53.

17.- Burr ML, Merrett TG, Dunstan FD, Maguire MJ: The development of allergy in high-risk children. Clin Exp Allergy 1997; 27 (11): 1247-53.

18.- Eggesbo M, Botten G, Stigum H, Samuelsen SO, Brunekreef B, Magnus $P$ : Cesarean delivery and cow milk allergy/intolerance. Allergy 2005; 60 (9): 1172-3.

19.- Raimondi F, Indrio F, Crivaro V, Araimo G, Capasso $L$, Paludetto $R$ : Neonatal hyperbilirubinemia increases intestinal protein permeability and the prevalence of cow's milk protein intolerance. Acta Paediatr 2008; 97 (6): 751-3.

20.- Sánchez-Valverde F, Gil F, Martínez D, Fernández B, et al: The impact of caesarean delivery and type of feeding on cow's milk allergy in infants and subsequent development of allergic march in childhood. Allergy 2009; 64 (6): 884-9.

21.- Acevedo Villafane C, Latorre Latorre F, Cifuentes Cifuentes L, Díaz-Martinez LA, Garza Acosta O: [Influence of breastfeeding and diet in the development of allergies in children]. Aten Primaria 2009; 41 (12): 675-80.

22.- Dioun AF, Harris SK, Hibberd PL: Is maternal age at delivery related to childhood food allergy? Pediatr Allergy Immunol 2003; 14 (4): 307-11.

23.- Kramer MS, Kakuma R: Optimal duration of exclusive breastfeeding. Cochrane Database Syst Rev 2002 (1): CD003517.

24.- Metsala J, Lundqvist A, Kaila M, Gissler M, Klaukka $T$, Virtanen SM: Maternal and perinatal characteristics and the risk of cow's milk allergy in infants up to 2 years of age: a case-control study nested in the Finnish population. Am J Epidemiol 2010; 171 (12): 1310-6.

25.- Tariq SM, Matthews SM, Hakim EA, Stevens M, Ars- 
had SH, Hide DW: The prevalence of and risk factors for atopy in early childhood: a whole population birth cohort study. J Allergy Clin Immunol 1998; 101 (5): 587-93.

26.- Vanto T, Juntunen-Backman K, Kalimo K, et al: The patch test, skin prick test, and serum milk-specific $\operatorname{IgE}$ as diagnostic tools in cow's milk allergy in infants. Allergy 1999; 54 (8): 837-42.

27.- Costa AJ, Sarinho ES, Motta ME, et al: Allergy to cow's milk proteins: what contribution does hypersensitivity in skin tests have to this diagnosis? Pediatr Allergy Immunol 2011; 22 (1 Pt 2): e133-8.

28.- Beauchamp JN, Gaboury I, Ni A, Boland MP, Mack DR: Solid-food introduction in infants diagnosed as having a cow's-milk protein-induced enterocolitis. J Pediatr Gastroenterol Nutr 2011; 52 (5): 639-43.

29.- Caffarelli C, Baldi F, Bendandi B, Calzone L, Marani $M$, Pasquinelli P: Cow's milk protein allergy in children: a practical guide. Ital J Pediatr 2010; 36: 5.

30.- Chaabane M, Bidat E, Chevallier B: [A new case of food protein-induced enterocolitis syndrome]. Arch Pediatr 2010; 17 (5): 502-6.

31.- Constantinide P, Trandafir LM, Burlea M: [The role of specific IgE to evolution and prognosis of cow's milk protein allergies in child]. Rev Med Chir Soc Med Nat Iasi 2011; 115 (4): 1012-7.

32.- de Seta L, Siani P, Cirillo G, Di Gruttola M, Cimaduomo L, Coletta $S$ : [The prevention of allergic diseases with a hypoallergenic formula: a follow-up at 24 months. The preliminary results]. Pediatr Med Chir 1994; 16 (3): 251-4.

33.- Denis M, Loras-Duclaux I, Lachaux A: [Cow's milk protein allergy through human milk.]. Arch Pediatr 2012 Jan 4.

34.- Dreborg $S$ : [Do breast milk and cow's milk hydrolysate protect against cow milk allergy or allergic disease?]. Lakartidningen 2006; 103 (20): 1627-8.

35.- Friedman NJ, Zeiger RS: The role of breast-feeding in the development of allergies and asthma. J Allergy Clin Immunol 2005; 115 (6): 1238-48.

36.- Halken $S$ : Prevention of allergic disease in childhood: clinical and epidemiological aspects of primary and secondary allergy prevention. Pediatr Allergy Immunol 2004; 15 Suppl 16: 4-5, 9-32.

37.- Katz Y, Goldberg MR, Rajuan N, Cohen A, Leshno M: The prevalence and natural course of food proteininduced enterocolitis syndrome to cow's milk: A largescale, prospective population-based study. J Allergy Clin Immunol 2011; 127 (3): 647-53 e3.

38.- Katz Y, Rajuan N, Goldberg MR, et al: Early exposure to cow's milk protein is protective against IgE-mediated cow's milk protein allergy. J Allergy Clin Immunol 2010; 126 (1): 77-82 e1.

39.- Lucarelli $S$, Di Nardo G, Lastrucci G, et al: Allergic proctocolitis refractory to maternal hypoallergenic diet in exclusively breast-fed infants: a clinical observation. BMC Gastroenterol 2011; 11: 82.

40.- Srinivasan P, Brandler M, D'Souza A, Millman P, Moreau $H$ : Allergic enterocolitis presenting as recurrent necrotizing enterocolitis in preterm neonates. J Perinatol 2010; 30 (6): 431-3.

41.- Thorsdottir I, Thorisdottir AV: Whole Cow's Milk in Early Life. Nestle Nutr Workshop Ser Pediatr Program 2011; 67: 29-40.

42.- Vandenplas Y, Veereman-Wauters G, De Greef E, et al: Gastrointestinal manifestation of cow's milk protein allergy or intolerance and gastrointestinal motility. J Pediatr Gastroenterol Nutr 2011; 53 Suppl 2: S15-7.

43.- von Berg A: Modified proteins in allergy prevention. Nestle Nutr Workshop Ser Pediatr Program 2009; 64: 239-47; discussion 47-57.

44.- Wal JM: Cow's milk proteins/allergens. Ann Allergy Asthma Immunol 2002; 89 (6 Suppl 1): 3-10.

45.- Yimyaem P, Chongsrisawat V, Vivatvakin B, Wisedopas $N$ : Gastrointestinal manifestations of cow's milk protein allergy during the first year of life. J Med Assoc Thai 2003; 86 (2): 116-23.

46.- Kristinsdottir H, Clausen M, Ragnarsdottir HS, et al: [Prevalence of food allergy in Icelandic infants during first year of life]. Laeknabladid 2011; 97 (1): 11-8.

47.- Leonard SA, Nowak-Wegrzyn A: Food protein-induced enterocolitis syndrome: an update on natural history and review of management. Ann Allergy Asthma Immunol 2011; 107 (2): 95-101; quiz, 62.

48.- Bruno G, Ziruolo MG, Lucenti P, Ziruolo G, Businco $L$ : [The primary and secondary prevention of allergic diseases in the child "at risk" for atopy: a review of the literature and the authors' personal experience]. Pediatr Med Chir 1995; 17 (3): 199-207.

49.- Cantani $A$ : Feeding high-risk infants with family history of allergy. Eur Rev Med Pharmacol Sci 1999; 3 (3): 143-6.

50.- Colombo ML, Cigolotti AC, Buschini MP, Erbeia M: [Prevention of intolerance to cow's milk protein in subjects at risk]. Minerva Pediatr 1987; 39 (20): 857-61.

51.- Kim J, Chang E, Han Y, Ahn K, Lee SI: The incidence and risk factors of immediate type food allergy during the first year of life in Korean infants: a birth cohort study. Pediatr Allergy Immunol 2011; 22 (7): 715-9.

52.- López Pérez G, Morfin Maciel BM, Huerta López J, et 
al: [Risk factors related to allergic diseases at Mexico City]. Rev Alerg Mex 2010; 57 (1): 18-25.

53.- Medeiros LC, Speridiao PG, Sdepanian VL, FagundesNeto U, Morais MB: [Nutrient intake and nutritional status of children following a diet free from cow's milk and cow's milk by-products]. J Pediatr (Rio J) 2004; 80 (5): 363-70.

54.- Agostoni C, Fiocchi A, Riva E, et al: Growth of infants with IgE-mediated cow's milk allergy fed different formulas in the complementary feeding period. Pediatr Allergy Immunol 2007; 18 (7): 599-606.

55.- Alexander DD, Schmitt DF, Tran NL, Barraj LM, Cushing CA: Partially hydrolyzed $100 \%$ whey protein infant formula and atopic dermatitis risk reduction: a systematic review of the literature. Nutr Rev 2010; 68 (4): 232-45.

56.- Allen KJ, Davidson GP, Day AS, et al: Management of cow's milk protein allergy in infants and young children: an expert panel perspective. J Paediatr Child Health 2009; 45 (9): 481-6.

57.- Dupont C, Chouraqui JP, de Boissieu D, et al: [Dietetic treatment of cow's milk protein allergy]. Arch Pediatr 2011; 18 (1): 79-94.

58.- Exl BM, Deland U, Secretin MC, Preysch U, Wall M, Shmerling $D H$ : Improved general health status in an unselected infant population following an allergen reduced dietary intervention programme. The ZUFFstudy-programme. Part I: Study design and 6-month nutritional behaviour. Eur J Nutr 2000; 39 (3): 89-102.

59.- Jin YY, Cao RM, Chen J, et al: Partially hydrolyzed cow's milk formula has a therapeutic effect on the infants with mild to moderate atopic dermatitis: a randomized, double-blind study. Pediatr Allergy Immunol 2011; 22 (7): 688-94. 